\title{
Grain-boundary-induced melting in quenched polycrystalline monolayers
}

\author{
Sven Deutschländer, ${ }^{1}$ Charlotte Boitard, ${ }^{1,2}$ Georg Maret, ${ }^{1}$ and Peter Keim ${ }^{1}$ \\ ${ }^{1}$ Department of Physics, University of Konstanz, 78464 Konstanz, Germany \\ ${ }^{2}$ ESPCI Paris Tech, 75005 Paris, France
}

\begin{abstract}
Melting in two dimensions can successfully be explained with the Kosterlitz-Thouless-Halperin-Nelson-Young (KTHNY) scenario which describes the formation of the high-symmetry phase with the thermal activation of topological defects within an (ideally) infinite monodomain. With all state variables being well defined, it should hold also as freezing scenario where oppositely charged topological defects annihilate. The Kibble-Zurek mechanism, on the other hand, shows that spontaneous symmetry breaking alongside a continuous phase transition cannot support an infinite monodomain but leads to polycrystallinity. For any nonzero cooling rate, critical fluctuations will be frozen out in the vicinity of the transition temperature. This leads to domains with different director of the broken symmetry, separated by a defect structure, e.g., grain boundaries in crystalline systems. After instantaneously quenching a colloidal monolayer from a polycrystalline to the isotropic fluid state, we show that such grain boundaries increase the probability for the formation of dislocations. In addition, we determine the temporal decay of defect core energies during the first few Brownian times after the quench. Despite the fact that the KTHNY scenario describes a continuous phase transition and phase equilibrium does not exist, melting in polycrystalline samples starts at grain boundaries similar to first-order phase transitions.
\end{abstract}

Since the seminal work of Peierls [1] and Mermin [2] on the existence of two-dimensional (2D) crystals, and the formulation of a microscopic melting theory by Kosterlitz, Thouless, Halperin, Nelson, and Young (KTHNY) [3-5], the equilibrium phase behavior of 2D crystalline systems has extensively been studied in experiment [6-16] and simulation [17-22]. The KTHNY theory predicts two consecutive continuous melting transitions from an infinite monocrystal to the isotropic fluid, which enclose a translationally short-range ordered but orientationally (quasi-)long-range ordered, so-called "hexatic" phase. The transitions are mediated by the dissociation of two kinds of topological defects: dislocations (an isolated pair of a five- and sevenfold coordinated particle) and disclinations (an isolated five- or sevenfold coordinated particle), at respectively different temperatures. While KTHNY melting is widely confirmed, the possibility of a strictly first-order scenario [23-27], an alteration [28-30], or a combination [31-33] of continuous and first-order characteristics has been discussed. A recent conclusion is that the precise melting mechanism depends on the type and range of the interaction potential [30]. In addition, the continuous two-step scenario might be preempted by one first-order transition. Either via the spontaneous creation of grain boundaries [24] if the (elastic) energy cost of a dislocation is lower than a critical value [28], by a simultaneous unbinding of dislocations and disclinations [34], or if the angular stiffness of the crystal is small enough [29]. Dislocations are the natural response of a crystal if the amplitude of an applied shear exceeds a critical value. The central ingredient of the KTHNY theory is the additional softening of the crystal due to the self-screening of thermally activated dislocation pairs, which is analytically included via a renormalization procedure $[4,5]$ : The creation and dissociation of dislocations towards the transition from the crystal to the hexatic fluid is enhanced in the presence of other dislocations. Compared to the mean-field ansatz, this results in a reduction of the melting temperature given by the fixpoint of the recursion relation of the renormalization procedure.
The screening of elasticity due to defects becomes crucial in a further sense if one considers large system sizes: Motivated by the Kibble-Zurek mechanism [35-37], a theory to predict the range of spatial correlations in symmetry breaking phase transitions under nonequilibrium conditions, it has recently been shown that the symmetry can only be broken locally at finite cooling rates, and domains with different orientation appear [38]. These are separated by domain walls which consist of strings of dislocations and disclinations, the respective monopoles (zero crossings) of the translational and orientational order parameter field. On the one hand, this is simply caused by causality. On the other, it is due to the fact that critical fluctuations are frozen out in the vicinity of the transition at any nonzero cooling rate. Thus, the preparation of an infinite monocrystal is an idealization and on large length scales one has to consider the presence of defect networks, e.g., grain boundaries consisting of chains of dislocations. The interaction between these boundaries and isolated defects in their vicinity is crucial for their mutual dynamic and the elastic and thermodynamic behavior in those regions. It has been suggested that an elastic deformation due to an applied stress on grain boundaries induces the creation of dislocation pairs [39] or the emission of partial dislocations [40] to release the stress. For atomic systems, it has been shown that grain boundaries can act as sources or sinks for point defects such as interstitials and vacancies [41-43], and that defect formation energies near grain boundaries are smaller than those in the bulk [44-46].

In three dimensions (3D), grain boundaries might also act as actuators for so-called premelting, which originally has been studied at surfaces or interfaces (see [47], and references therein). In principle, premelting occurs when at temperatures smaller than the melting temperature $T_{m}$, the system can lower its free energy by replacing the grain boundary by two crystal-liquid interfaces, while the width of this liquid layer then diverges towards the melting point. In lattice-gas models [48] and Lennard-Jones systems [49], a structural 
transition to a disordered, liquidlike structure inside or near the grain boundary has been observed well below $T_{m}$. Subsequent simulations show that systems with grain boundaries cannot be superheated [50] and that boundaries induce nucleation of the melting process in their vicinity [51]. Experimentally, premelting has intensively been studied using colloidal crystals where single particle resolution allows one to resolve the structure, dynamics, and defect configurations on a "microscopic" scale. For 3D polycrystals, the dynamics of particles in the vicinity of grain boundaries is largely enhanced in advance of the melting transition: The liquid nucleates at the domain interface [52]. For multilayer polycrystalline films, this effect is found to dominate as long as the film thickness is not too small (e.g., $>4$ layers), and the systems melts via the generation and expansion of "liquid stripes" initiated from the grain boundaries [53]. As the layer thickness is further reduced, melting is also induced by liquid nucleation within crystalline domains due to the creation of defect clusters, and then preferably continues to the spatially random excitation of isolated defects [53]. This weakening of premelting is not unexpected approaching the monolayer case in 2D where nucleation is more and more suppressed and the melting mechanism is expected to be continuous and KTHNY-like. Then, interfacial energies might become irrelevant and signatures of premelting are entirely caused by the enhanced self-screening of topological defects near grain boundaries.

Previous thermodynamic studies in simulations and colloidal experiments have been conducted under equilibrium or quasi-equilibrium. However, systems often are subject to nonequilibrium conditions due to local or global rapid quenches of the control parameter. In this experimental work, we carry those factors to a limit, by quenching colloidal monolayers from states with different degrees of polycrystallinity into the isotropic fluid via an almost infinite heating rate. We analyze the formation probabilities of defects (their so-called fugacity) as a function of distance to the initial grain boundaries and as a function of time after the quench of the thermodynamic order parameter. We show that compared to the bulk (the center of the crystalline domains), the defect creation is enhanced in the vicinity of grain boundaries. The long distance behavior of those probabilities allows us to determine the time evolution of the bulk core energies within the first few Brownian times after the onset of the quench.

Our experimental system consists of a colloidal suspension of superparamagnetic polystyrene beads with a diameter $\sigma \approx$ $4.5 \mu \mathrm{m}$ and a mass density of $1.7 \mathrm{~kg} / \mathrm{dm}^{3}$, which is confined to two dimensions by a bottom glass plate and gravity. The whole monolayer contains $\sim 10^{5}$ particles, and a subwindow of $1 \mathrm{~mm}^{2}$ with $\approx 5900$ particles is tracked with a time resolution of $\approx 1.7 \mathrm{~s}$, which is well below the Brownian time scale $\tau_{B} \approx$ $172 \mathrm{~s}$ [54]. A repulsive dipole-dipole interaction is induced by an external magnetic field $B$ applied perpendicular to the colloidal monolayer. The magnetic field tunes the crucial order parameter, the ratio of mean magnetic energy of two particles and thermal energy:

$$
\Gamma=\frac{E_{\mathrm{mag}}}{k_{B} T}=\frac{\mu_{0}(\pi n)^{3 / 2}(\chi B)^{2}}{4 \pi k_{B} T},
$$

where $\mu_{0}$ is the vacuum permeability and $\chi \approx 1.9 \times$ $10^{-11} \mathrm{Am}^{2} / \mathrm{T}$ the magnetic susceptibility per particle. Temperature $T$ and the 2D particle density $n$ are kept constant (room temperature and a mean particle distance of $a_{0}=n^{-1 / 2} \approx$ $12.9 \mu \mathrm{m}) . \Gamma$ acts like an inverse temperature $\left(\propto T^{-1}\right)$ and can be tuned homogeneously within the sample, which excludes melting from the surface of the system. At large values of $\Gamma$ ("low temperature") the system is in a crystalline state with (local) triangular symmetry, while at small $\Gamma$ values ("high temperature") the isotropic fluid is maintained. For equilibrium conditions, melting temperatures are found at $\Gamma_{m} \approx 70.3$ for the crystal-hexatic transition, and $\Gamma_{i} \approx 67.3$ for the hexatic-isotropic transition [16]. The instantaneous quench is conducted from two initially prepared, polycrystalline samples $A$ and $B$ at $\Gamma_{I} \approx 100$ (Fig. 1) to a final value
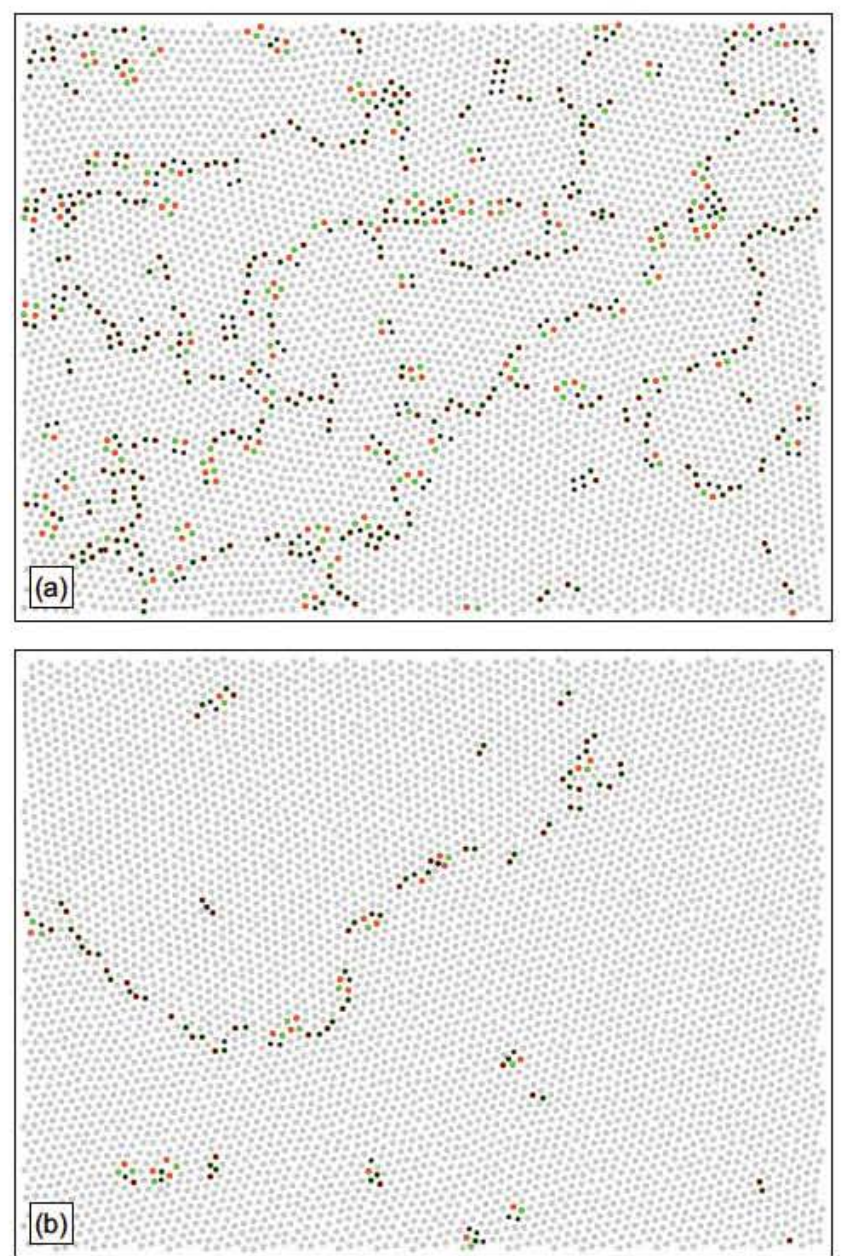

FIG. 1. (Color online) Illustration of the initial defect configuration of the two polycrystalline samples $A$ (a) and $B$ (b) $\left(1 \mathrm{~mm}^{2}\right.$, $\approx 5900$ particles). Particles with six nearest neighbors (sixfold coordinated) are colored gray (bright), five- and sevenfold coordinated particles orange and green, and particles with less than five or more than seven nearest neighbors blue (dark). While sample $A$ is in initial state of high polycrystallinity, sample $B$ consists of two large monocrystalline domains separated by one high-angle grain boundary and some isolated, frozen-in defects. Particles additionally indicated with smaller black dots are defects for more than $50 \%$ of the time before the polycrystalline structure is quenched to the isotropic fluid. 


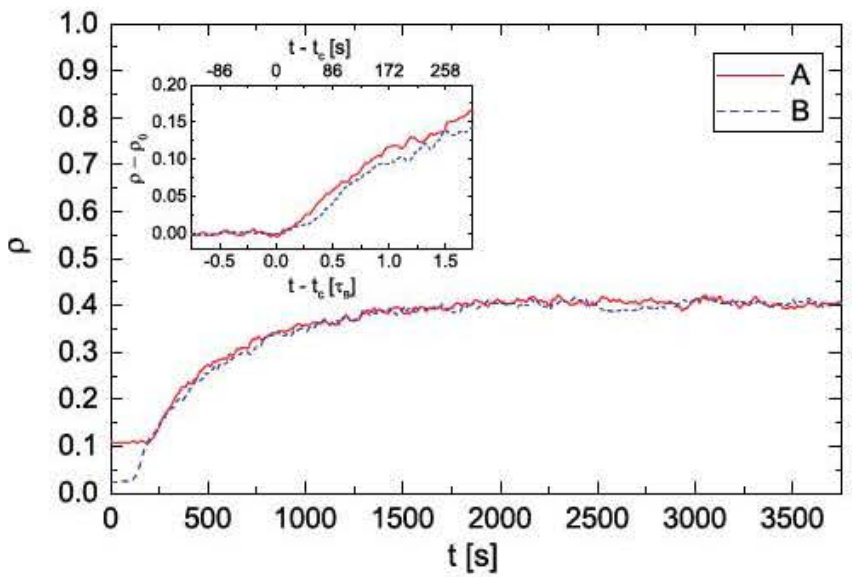

FIG. 2. (Color online) Time evolution of the defect density $\rho$ for samples $A$ (solid line) and $B$ (dashed line) shortly before and after an instantaneous quench (around $\approx 150 \mathrm{~s}$ ) from the polycrystal to the isotropic fluid. The samples start at different initial defect densities $\rho_{0}$ and approach a common value at large times which is a function of the final value of the control parameter $\Gamma$. The inset shows the reduced density $\rho-\rho_{0}$ shifted with respect to the onset of the quench to $t-t_{c}$.

of $\Gamma_{F} \approx 27$. While sample $A$ is in an initial state of high polycrystallinity, sample $B$ consists of two large domains, separated by a large-angle grain boundary. These states were created by driving the system from the isotropic fluid to the crystalline state with different finite cooling rates (fast rate for sample $A$ and slow rate for sample $B$ ), which results in different kinds of polycrystallinity and different domain size distributions [38].

In $2 \mathrm{D}$, all particles being not sixfold coordinated belong to a defect, and the defect density $\rho$ is given by the number of defects divided by the total number of particles $N$. We define the initial grain boundaries in the following way: Every particle which is a defect more than $50 \%$ of the time in the steady polycrystalline state (before the system is quenched at $t_{c}$ ), is considered as a grain boundary particle or a "frozen" defect (particles with smaller black dots in Fig. 1) [55]. This grain boundary criteria does not capture all defects at any given time step before the quench to account for the creation and annihilation of virtual dislocation pairs, which have a short lifetime. The defect density as a function of temperature is well known in equilibrium: From a plateau in the crystal the defect density $\rho$ performs a sharp (but still continuous) increase across the two melting transitions while in the isotropic fluid it increases only gradually [16]. The evolution of $\rho$ under the instantaneous quench into the isotropic fluid is shown in Fig. 2: After the quench, $\rho$ increases sharply from an initial defect density $\rho_{0}(\approx 0.11$ for sample $A$ and $\approx 0.03$ for sample $B)$ and approaches a finite value at large times $(\approx 0.4$ at $3750 \mathrm{~s})$ equal for both samples [56]. $\rho_{0}$ is calculated from a time average of $\rho$ within $\tau_{B}$ directly before the quench: $\rho_{0}=\left\langle\rho\left(t<t_{c}\right)\right\rangle_{t}$. The inset shows the reduced density $\rho-\rho_{0}$, which emphasizes the evolution of thermally activated defects with respect to the onset of the quench.

To study the spatial effect of grain boundaries, we classify particles into equidistant intervals of $10 \mu \mathrm{m}$ according to their (smallest) distance $x$ to the initially frozen defects, and

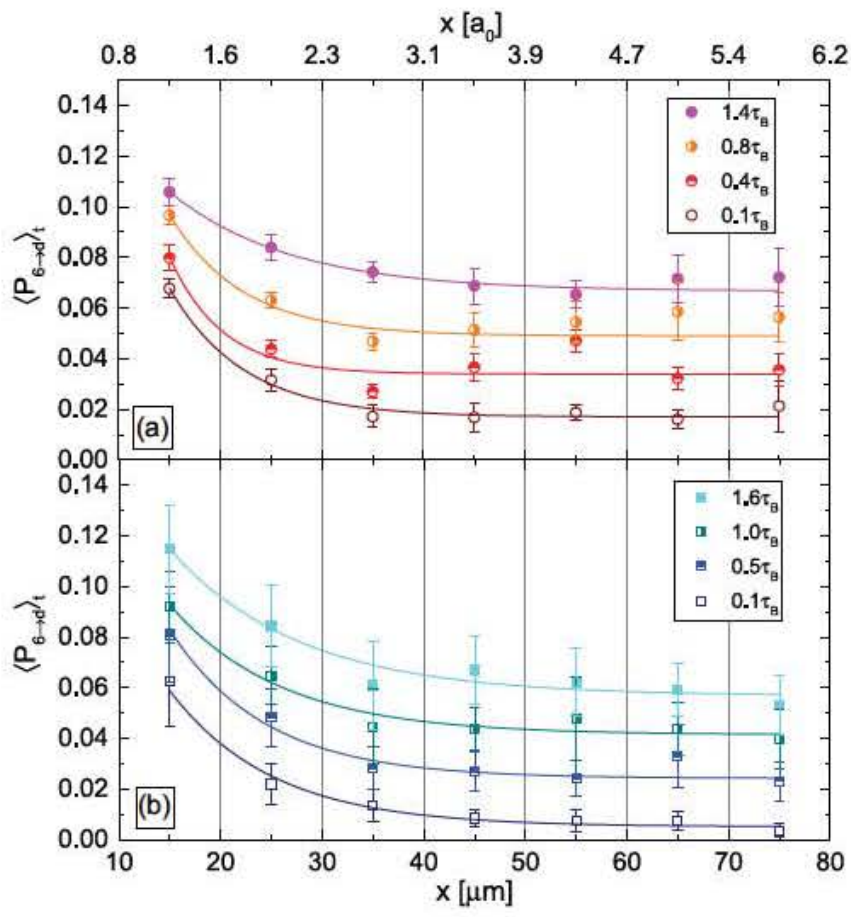

FIG. 3. (Color online) Time averaged formation probabilities $\left\langle P_{6 \rightarrow d}\right\rangle_{t}$ of defects for sample $A$ (a) and $B$ (b), as a function of distance $x$ to grain boundaries and within the first Brownian times. The average is performed within an interval of $\pm 0.1 \tau_{B}$ and error bars are standard deviations $( \pm \sigma)$ from the time average. Probabilities are enhanced close to the grain boundaries, decay towards the bulk (middle of crystalline domains), and raise as a function of time. Solid lines are exponential fits.

determine the respective probabilities of defect formation after the onset of the quench. More precisely, we calculate the time dependent conditional probability that a sixfold coordinated particle becomes nonsixfold coordinated after a tenth of the Brownian time $\left(0.1 \tau_{B} \approx 17 \mathrm{~s}\right)$ has passed:

$$
P_{6 \rightarrow d}(t)=P\left(\text { defect at } t+0.1 \tau_{B} \mid \text { nondefect at } t\right) .
$$

To obtain a discriminable time evolution of the spatial classification within the first Brownian times, we perform a time average of those probabilities within intervals of \pm 10 time steps $\left(\approx 0.1 \tau_{B}\right)$. The results are shown Fig. 3 as a function of distance $x$ to grain boundaries, where different curves correspond to different times after the onset of the quench $t_{c}$. The effect of grain boundaries on the melting behavior can clearly be seen: Close to the boundaries, $\left\langle P_{6 \rightarrow d}\right\rangle_{t}$ is enhanced for both samples. Within the first couple of mean particle distances, the probabilities decay to a finite value $P_{0}$. This is reasonable since even with a vanishing influence of grain boundaries, the creation probability of virtual dislocation pairs should remain finite. In equilibrium melting, a discrete energy is needed for the formation of a single defect which is called the core energy $E_{c}$. The defect formation probability (or fugacity) $P_{0}$ is a function of that energy and the temperature: $P_{0}=$ $e^{-E_{c} / k_{B} T}$. The data shows that the bulk fugacities gradually increase with time, even if the interaction strength is reduced instantaneously and remains constant after $t_{c}$. This is due to the fact that the defect core energy is a function of the elastic 
constants and therefore depends on the structure of the system, but the structural changes delay relative to the rapidly changed control parameter. The structural evolution is accompanied by changes in the correlation length which is directly coupled to the relaxation time. This delay in the evolution of the correlation length (with respect to the equilibrium behavior) is the same effect responsible for the common Kibble-Zurek mechanism. Here, it can be seen as an inverse version of it (e.g., studied for two-dimensional superfluids [57] or trapped Bose gases [58]) with an infinite heating rate and for the case of an initial state of locally broken symmetry.

To extract the time dependent core energies in the bulk, we fit the probabilities with an exponential $P(x)=a e^{-x / b}+$ $P_{0}$ (solid lines in Fig. 3). The values of $P_{0}$ as a function of time (after the onset of the quench) are shown in Fig. 4 for both samples: Within the first Brownian time, $P_{0}(t)$ increases linearly, and seems to slightly saturate at larger times. This can be expected towards the attainment of the isotropic structure. The inset shows the respective time dependent (relative) defect core energies $E_{c}(t) / k_{B} T$. After the onset of the quench, they decay from $\approx 4.1 k_{B} T$ for sample $A$ and $\approx 5.3 k_{B} T$ for sample $B$ to a similar value around $2.7 k_{B} T$ for both samples. For the weak polycrystalline sample $(B)$, we find that the defect core energy shortly after the quench is very similar to the core energy of isolated disclinations $E_{c}=5.5 k_{B} T$ slightly above the hexatic-isotropic melting temperature under equilibrium conditions [59]. That the defect core energy for sample $A$ is smaller than for sample $B$ can be addressed to its higher degree of polycrystallinity. From linear fits $P_{0}(t)=a t+b$ to the bulk probabilities (solid lines in Fig. 4) we can extract the short-time behavior of the core energies and find $E_{c}(t) / k_{B} T=$ $-\ln [0.00046(2) t+0.013(1)]$ for sample $A$ and $E_{c}(t) / k_{B} T=$ $-\ln [0.00040(3) t+0.002(1)]$ for sample $B$.

The effect of grain boundaries on melting transitions is debated for different systems: in 2D as an alternative scenario to the KTHNY theory via grain boundary formation in a monocrystal, preceding the thermal excitation and dissociation of dislocation pairs $[24,28]$ and in $3 \mathrm{D}$ as the nucleation of the melting process at grain boundaries in initially polycrystalline states; so-called premelting $[48,49,51,52]$. While KTHNY melting starts with an infinite, defect-free monocrystal, the Kibble-Zurek mechanism shows that for continuous transitions the domain size is in principle limited by the cooling

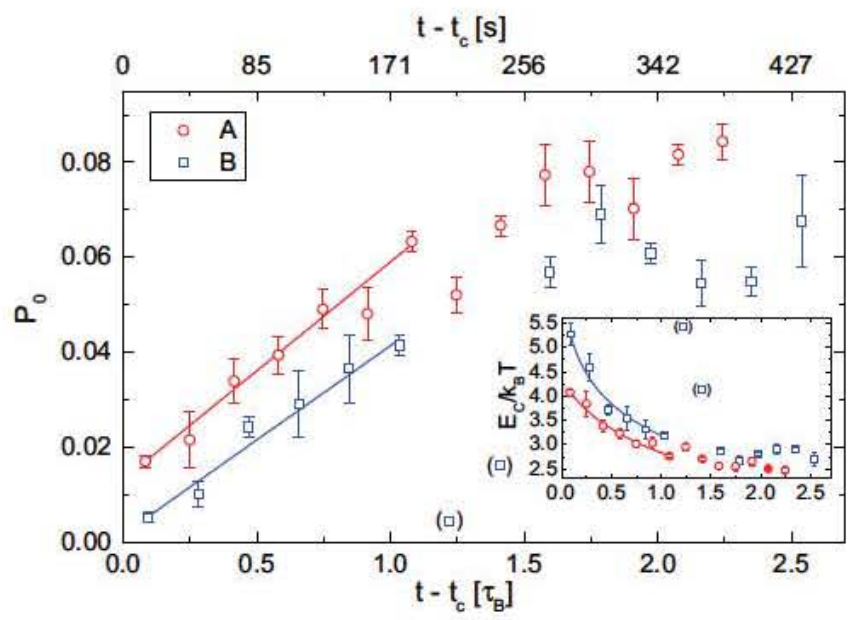

FIG. 4. (Color online) Time evolution of bulk defect probabilities $P_{0}$ and respective defect core energies $E_{c}$ (inset) after the onset of the quench $\left(t_{c}\right)$ for both samples. Error bars are standard deviations $( \pm \sigma)$ of the fit parameter $P_{0}$. At short times, we observe a linear time evolution for $P_{0}$ (solid lines are linear fits to $P_{0}$ ) and core energies decay at large times. Bracketed data points denote time intervals where the exponential fit $P(x)$ failed due to too large fluctuations of $\left\langle P_{6 \rightarrow d}\right\rangle_{t}$.

rate [35-37]. Slow cooling rates serve large domains, while fast cooling rates lead to small domains. Thus, beside the discussion of thermally induced grain boundary melting, frozen-in grain boundaries will naturally be present in real systems. We show that the presence of those grain boundaries enhances the melting process: The fugacity is significantly increased in the vicinity of the boundary. One has to note that this should not be interpreted in terms of premelting of 3D systems, simply since the underlying phase equilibrium or a surface tension of distinct phases does not exist. In addition we find that the fugacity towards the "bulk" is affected by the degree of polycrystallinity and decreases logarithmically at short times.

P.K. gratefully acknowledges financial support from the Deutsche Forschungsgemeinschaft (KE 1168/8-1).
[1] R. Peierls, Helv. Phys. Acta 7, 81 (1934); Ann. Inst. Henri Poincaré 5, 177 (1935).

[2] N. D. Mermin, Phys. Rev. 171, 272 (1968).

[3] J. M. Kosterlitz and D. J. Thouless, J. Phys. C 5, L124 (1972); 6, 1181 (1973).

[4] B. I. Halperin and D. R. Nelson, Phys. Rev. Lett. 41, 121 (1978); D. R. Nelson and B. I. Halperin, Phys. Rev. B 19, 2457 (1979).

[5] A. P. Young, Phys. Rev. B 19, 1855 (1979).

[6] A. Widom, J. R. Owers-Bradley, and M. G. Richards, Phys. Rev. Lett. 43, 1340 (1979).

[7] W. F. Brinkmann, D. S. Fisher, and D. E. Moncton, Science 217, 693 (1982).

[8] T. F. Rosenbaum, S. E. Nagler, P. M. Horn, and R. Clarke, Phys. Rev. Lett. 50, 1791 (1983).
[9] R. E. Kusner, J. A. Mann, J. Kerins, and A. J. Dahm, Phys. Rev. Lett. 73, 3113 (1994).

[10] K. Zahn, R. Lenke, and G. Maret, Phys. Rev. Lett. 82, 2721 (1999).

[11] K. Zahn and G. Maret, Phys. Rev. Lett. 85, 3656 (2000).

[12] D. E. Angelescu, C. K. Harrison, M. L. Trawick, R. A. Register, and P. M. Chaikin, Phys. Rev. Lett. 95, 025702 (2005).

[13] A. V. Petukhov, D. van der Beek, R. P. A. Dullens, I. P. Dolbnya, G. J. Vroege, and H. N. W. Lekkerkerker, Phys. Rev. Lett. 95, 077801 (2005).

[14] P. Keim, G. Maret, and H. H. von Grünberg, Phys. Rev. E 75, 031402 (2007).

[15] Y. Han, N. Y. Ha, A. M. Alsayed, and A. G. Yodh, Phys. Rev. E 77, 041406 (2008). 
[16] S. Deutschländer, A. M. Puertas, G. Maret, and P. Keim, Phys. Rev. Lett. 113, 127801 (2014).

[17] D. Frenkel and J. P. McTague, Phys. Rev. Lett. 42, 1632 (1979).

[18] K. J. Strandburg, S. A. Solla, and G. V. Chester, Phys. Rev. B 28, 2717 (1983).

[19] K. Chen, T. Kaplan, and M. Mostoller, Phys. Rev. Lett. 74, 4019 (1995).

[20] S. Z. Lin, B. Zheng, and S. Trimper, Phys. Rev. E 73, 066106 (2006).

[21] S. Prestipino, F. Saija, and P. V. Giaquinta, Phys. Rev. Lett. 106, 235701 (2011).

[22] K. Wierschem and E. Manousakis, Phys. Rev. B 83, 214108 (2011).

[23] T. V. Ramakrishnan, Phys. Rev. Lett. 48, 541 (1982).

[24] S. T. Chui, Phys. Rev. Lett. 48, 933 (1982).

[25] H. Kleinert, Phys. Lett. A 95, 381 (1983).

[26] S. Toxvaerd, Phys. Rev. Lett. 44, 1002 (1980).

[27] F. F. Abraham, Phys. Rev. Lett. 44, 463 (1980)

[28] Y. Saito, Phys. Rev. Lett. 48, 1114 (1982).

[29] H. Kleinert, Phys. Lett. A 136, 468 (1989).

[30] S. C. Kapfer and W. Krauth, Phys. Rev. Lett. 114, 035702 (2015).

[31] A. H. Marcus and S. A. Rice, Phys. Rev. Lett. 77, 2577 (1996).

[32] E. P. Bernard and W. Krauth, Phys. Rev. Lett. 107, 155704 (2011).

[33] S. Prestipino, F. Saija, and P. V. Giaquinta, J. Chem. Phys. 137, 104503 (2012)

[34] K. J. Strandburg, Rev. Mod. Phys. 60, 161 (1988).

[35] Ya. B. Zel'dovich, I. Yu. Kobzarev, and L. B. Okun', Zh. Eksp. Teor. Fiz. 67, 3 (1974) [Sov. Phys.-JETP 40, 1 (1975)].

[36] T. W. B. Kibble, J. Phys. A 9, 1387 (1976); Phys. Rep. 67, 183 (1980).

[37] W. H. Zurek, Nature (London) 317, 505 (1985); Acta Phys. Pol. B 24, 1301 (1993).

[38] S. Deutschländer, P. Dillmann, G. Maret, and P. Keim, Proc. Natl. Acad. Sci. U.S.A. 112, 6925 (2015).

[39] W. T. Read and W. Shockley, Phys. Rev. 78, 275 (1950).

[40] H. Van Swygenhoven, P. M. Derlet, and A. Hasnaoui, Phys. Rev. B 66, 024101 (2002).
[41] R. W. Balluffi, Y. Komem, and T. Schober, Surf. Sci. 31, 68 (1972).

[42] M. R. Sørensen, Y. Mishin, and A. F. Voter, Phys. Rev. B 62, 3658 (2000).

[43] B. Wang, Y. Puzyrev, and S. T. Pantelides, Carbon 49, 3983 (2011).

[44] H.-S. Lee, T. Mizoguchi, J. Mitsui, T. Yamamoto, S. J. L. Kang, and Y. Ikuhara, Phys. Rev. B 83, 104110 (2011).

[45] M. A. Tschopp, M. F. Horstemeyer, F. Gao, Y. Sun, and M. Khaleel, Scr. Mater. 64, 908 (2011).

[46] X.-M. Bai, L. J. Vernon, R. G. Hoagland, A. F. Voter, M. Nastasi, and B. P. Uberuaga, Phys. Rev. B 85, 214103 (2012).

[47] J. G. Dash, Rev. Mod. Phys. 71, 1737 (1999).

[48] R. Kikuchi and J. W. Cahn, Phys. Rev. B 21, 1893 (1980).

[49] G. Ciccotti, M. Guillopé, and V. Pontikis, Phys. Rev. B 27, 5576 (1983).

[50] J. Q. Broughton and G. H. Gilmer, Phys. Rev. Lett. 56, 2692 (1986).

[51] S. R. Phillpot, J. F. Lutsko, D. Wolf, and S. Yip, Phys. Rev. B 40, 2831 (1989).

[52] A. M. Alsayed, M. F. Islam, J. Zhang, P. J. Collins, and A. G. Yodh, Science 309, 1207 (2005).

[53] Y. Peng, Z. Wang, A. M. Alsayed, A. G. Yodh, and Y. Han, Phys. Rev. Lett. 104, 205703 (2010).

[54] We define the Brownian time scale as $\tau_{B}:=(\sigma / 2)^{2} / D_{0}$ where the short-time diffusion constant $D_{0} \approx 0.0295 \mu \mathrm{m}^{2} / \mathrm{s}$ is observed from equilibrium measurements of the isotropic fluid state.

[55] We analyzed our data also with smaller and larger threshold values but did not see a significant difference in the results.

[56] See Supplemental Material at http://link.aps.org/supplemental/ 10.1103/PhysRevE.92.060302 for a movie of the defect dynamics in sample $A$. Initially frozen-in grain boundaries (and defects) are illustrated with black dots throughout the movie.

[57] L. Mathey and A. Polkovnikov, Phys. Rev. A 81, 033605 (2010).

[58] V. I. Yukalov, A. N. Novikov, and V. S. Bagnato, Phys. Lett. A 379, 1366 (2015).

[59] C. Eisenmann, U. Gasser, P. Keim, G. Maret, and H. H. von Grünberg, Phys. Rev. Lett. 95, 185502 (2005). 